\title{
Vehicle CALS - results of a big challenge to virtual development
}

\author{
T. Mase \\ Nissan Digital Process LTD. \\ Atsugi- Aida Bldg., \\ 2-9-6, Nakacho, Atsugi-City, Kanagawa, 243-0018, Japan \\ Telephone: +81-462-25-3923 \\ Facsimile: +81-462-22-6011 \\ E-mail:mase@dipro.co.jp
}

\begin{abstract}
A major issue for the automotive industry is the shortening of the time required to develop new vehicles. A very effective way of solving this problem is changing to a setup where development processes are based on digital information. To this end, companies are taking positive steps and using information technology (IT) to enable reforms to processes.

Understanding the limits to the effectiveness of this strategy if each company merely carries out its own activities, the year before last, the Ministry of International Trade and Industry (MITI) established of the V-CALS Consortium with the cooperation of the automotive and information industries. This project implemented demonstrations and research into such matters as the promotion of standardization for the development of basic information technology and for information distribution, both of which are required to enable digital processes to function. As a result, the project was able to confirm the effectiveness of digital processes and provide recommendations regarding the prerequisites to the formation of digital processes (for example, new generation PDM).
\end{abstract}

\section{Keywords}

Vehicle CALS, digital process, CAD/CAM, DMU, PDM, CORBA, STEP, EDI, SGML 


\section{AIMS OF V-CALS}

In the processes from vehicle planning to preparation for production, V-CALS aims to minimize the amount of trial manufacture in the development stage, and to enable work processes, that is "digital processes" in which products can be directly produced in a world without trial manufacture and paper drawings. To this end VCALS has:

1) clarified the problems and issues relating to the current situation that are impeding digitalization, and

2) clarified the use and requirements of a common information system base that can solve such issues the problems

V-CALS is also developing new generation information systems in appropriate fields and implementing demonstrations and research into the effectiveness of new work setups.

\section{OVERVIEW OF V-CALS}

To enable the above aims to be realized, in May 1996, 32 companies including five automotive companies, five information companies, and 22 parts suppliers set up a consortium. Its actual activities were divided into four areas for which separate working groups (WG1 through WG4) were established to carry out the following demonstrations and research.

WG1 was further divided into two subgroups (SG11 and SG12). SG11 used the most advanced current technology to trial digital processes and clarified the prerequisites for enabling the processes. Parallel to this, SG12 conducted research into the tools (PDM) needed to enable the actual new generation of digital processes to be realized in a way that fulfilled the prerequisites provided by SG11.

WG2, WG3, and WG4 implemented demonstrations of standards that form the basis of digital processes, set standards, and developed and evaluated translators.

Specifically, WG2 conducted demonstrations in the application of AP203 and AP214 in STEP. WG3 researched the application of the industry standard EDI for receiving orders for mass production. WG4 was divided into two subgroups. SG41 converted motor vehicle maintenance manuals into SGML, and SG42 placed all types of national regulations and information relating to motor vehicles onto a database, and demonstrated systematization, standardization, and application. A diagram showing the working groups and their functions is given in Figure 1.

The results of the activities of each group are reported below. 


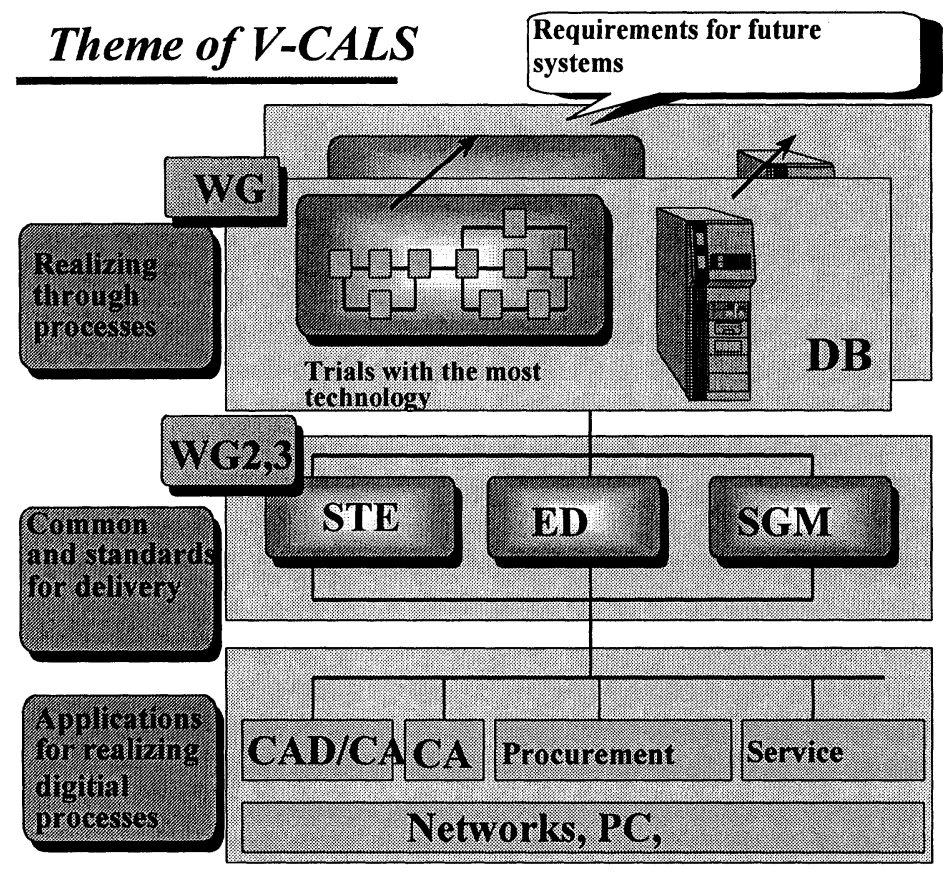

Figure.1 Functions of V-CALS

\section{TRIAL OF DIGITAL PROCESS (WG1/SG11)}

\subsection{Purpose}

The purposes of the trials are to apply current CALS technology into business processes related to the development of motor vehicles and to clarify the effectiveness of digital processes and issues relating to them(Figure.2). In the demonstrations, a virtual enterprise was created using participating companies and focusing on all phases from the development of vehicles to the preparation for their production. Using the most advanced current IT, the group clarified how far current technology would go in developing vehicles in a virtual world and extracted the problems that remained (gap between virtual and real worlds). 


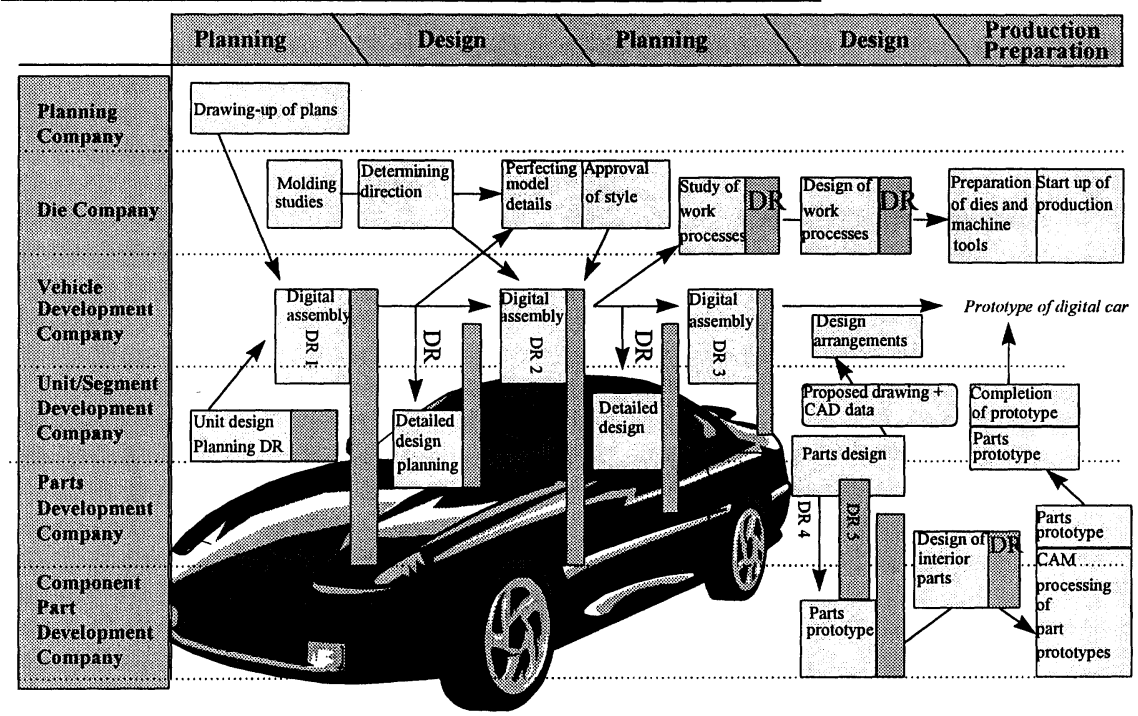

Figure.2 Digital Process

\subsection{Overview of Trials}

Trials were undertaken for the following three reasons:

1) To verify the effectiveness of digital mock-ups and extract related issues In the digital process, all parts in a motor vehicle were expressed as threedimensional models in a computer. These were used for design investigation. The three-dimensional digital models that were used instead of drawings are called digital mock ups (DMU). The group then examined how useful these DMUs were as replacements for actual vehicles.

2) To verify the effectiveness of electronic conferences and extract related issues Electronic conferences and electronic design reviews overcome the restrictions of time and space. Attempts were made to determine how effective they were in reducing development periods. To this end, asynchronous and synchronous electronic conferences were held using a wide-band network, their effectiveness tested and related issues clarified. To verify the effectiveness of digital management and extract related issues

3) Delivery management, progress management, and design change management in the virtual enterprise was conducted based on digital information. The effectiveness of digital management was tested and related issues extracted. 


\subsection{Results of Trial}

(1) Digital mock up trial

Testing of functions for expressing "product appearance"

The functions used to create digital clay models for conceptual designs were basically effective. For a particular amount of work and time a full-size clay model of the same quality could be made for the same type of conceptual design. In particular, in the initial design stage where details are not shaped and where investigation of the conceptual design is not particularly stringent, digital clay models could be made efficiently in a short time using these functions.

However, because the modeling methods used in these functions are analytical (planar construction must be reduced to cross-sectional level), they are not suitable for use (are not intuitive) in conceptual design where overall vague creativity is required.

It is also difficult to investigate a detail design when the precision of a life-size model is provided and use after the intermediate stage of design is not practical.

Editing functions such as surface connection, cut, redraw, copy, and partial change are simple to use, are precise and enable trial and error of digital clay models. This was seen as the strength of the digital clay models. However, the partial change function was, compared to the clay model, not free or flexible enough for design work where impromptu trial and error is common. It would be easier to remake the model than to use this function.

When there are many trimmed surfaces, the amount of data increases and becomes difficult to handle.

Testing of functions for expressing "three-dimensional form"

The effectiveness of creating and editing functions for digital models of curved surfaces was tested.

Forms were recognized and differentiating functions were very effective. The legibility of form was better than in drawings.

Adequate functions for editing three-dimensional forms and data are provided and there are no problems with ease of operation, reliability, and response.

In the drawing of cross-sectional forms, a great deal of work is required in paper drawing and there is a great difference in the precision of such drawings as produced by experienced and non-experienced persons. The digital functions however are able to provide highly accurate cross-sectional drawings in threedimensional forms for complex parts, regardless of the operator.

Note however, that editing greatly impacts the process in which threedimensional data is created and the work involved in editing models is disproportionate to the scale of the design changes and so, in may cases, there is reliance on the data creation process. In practice, a lengthy training period will be required to enable efficient editing. 
Testing of effectiveness of functions for expressing the "operation of multiple parts".

We tested the effectiveness of writing and editing functions for digital models of moving parts.

We were able to test the effectiveness of functions for expressing the "operation of multiple parts" and found that it was possible to design the mobile footprints required in the design of mobile parts. However, where restrictive conditions are defined for models, functions that can directly revise design without having to be concerned about the deletion of restricting conditions, changes to form, and definition of restricting conditions are required. Other functions required are those that enable easy visualization of restricting conditions.

When digitalization takes place, even when the scale of a model changes, such as when in the past a clay model had to be re-created, the functions can respond with flexibility and speed, conceptual trial and error can be checked simply, positioning between parts can be intuitively understood and any interference simply detected, and models can be quickly tested by people associated with the next process or people in non-design departments. The effectiveness of these was acknowledged.

On the other hand, there are a great many limitations to the digital process including a lack of functionality prerequisites, the amount of work involved with digitalization, the load of excessive data, and the load on the equipment.

In the primary demonstration, verified technology was used as the required technology and so the first step in the digital process, that is digitalization of the "product" was done almost perfectly with current technology.

However, problems start to mount when we look at the practicality of digitalized information, in particular when the field of vision is extended to include the sharing of information between corporations - that is the CALS concept.

\section{(2) Electronic conference trial}

Meetings that would conceivably occur during the digital processes, such as meetings for instructions, making contacts, conferencing, and reviewing, were conducted using a combination of synchronous (video conferencing using PCs), asynchronous (document based conferencing using electronic notice boards), and mobile conferencing. The effectiveness of such conferences was evaluated and technical problems extracted.

(3) Digital management trial

We report on the results of the trial of design change management, the central theme in the digital management demonstration. In this demonstration, we used the "production preparation design change function" and "parts design change function" developed for this demonstration to digitalize the preparation for production work of motor vehicle manufacturers and the design and preparation for production work of parts manufacturers. The following demonstrations were held, 
and their effectiveness evaluated.

The results showed reduction in time required to deal with issues and a reduction in the work involved. This resulted in increased productivity.

Specifically, there were variation depending on the different stages of the work but nevertheless, the work involved in follow-ups between jobs was reduced by half.

Also, the work flow was visualized in the evaluation. This enabled the processes to adhere to rules and reduced duplication. Furthermore, work management was made more efficient by linking management of product information to the work flow. Redundant work time was eliminated and work results were more uniform.

As shown above, digitalization of the parts design and preparation for production work enables work to be automatically received on the network and enables more efficient management of the entire work flow. The effectiveness of this procedure met with approval.

\subsection{SG11 Summary}

The primary demonstration enabled an evaluation of the theoretical effectiveness of digital processes, centered around digital mock ups. In addition, current problems were clarified. This is an important step towards realization of digital processes.

\section{SG12 - NEXT GENERATION PDM RESEARCH \\ 4.1 Aims of the SG12}

To halve development time, the shape of future digital processes was mapped out and requisites for a new generation of PDM extracted. Prototypes were developed and demonstrations implemented using basic IT. The practicality of these was tested.

\subsection{Next Generation Development Processes and PDM}

In the development of vehicles the development processes, from initial product planning through to preparation for production, traditionally have overlaps in the sequential flow. However, in digital processes that use data, in all digital development stages of the digital design audit, digital mock up, digital laboratory, and the digital factory, everything can be studied in an interactive manner using data definitions and growth. Therefore, the existence of a so-called digital car where everything can be defined digitally is important. This car is known as the product object of a motor vehicle. Essential elements for the progress of development, which will enable this product object to evolve and grow, have been identified as cooperative design and co-evolution management. We therefore conceived a new generation of PDM that would enable response to the large scale global distribution that is characteristic of automotive development and that would provide a multi-dynamic view in which all sorts of knowledge will be accumulated 


\section{WG1/SG12: Next-generation PDM research}

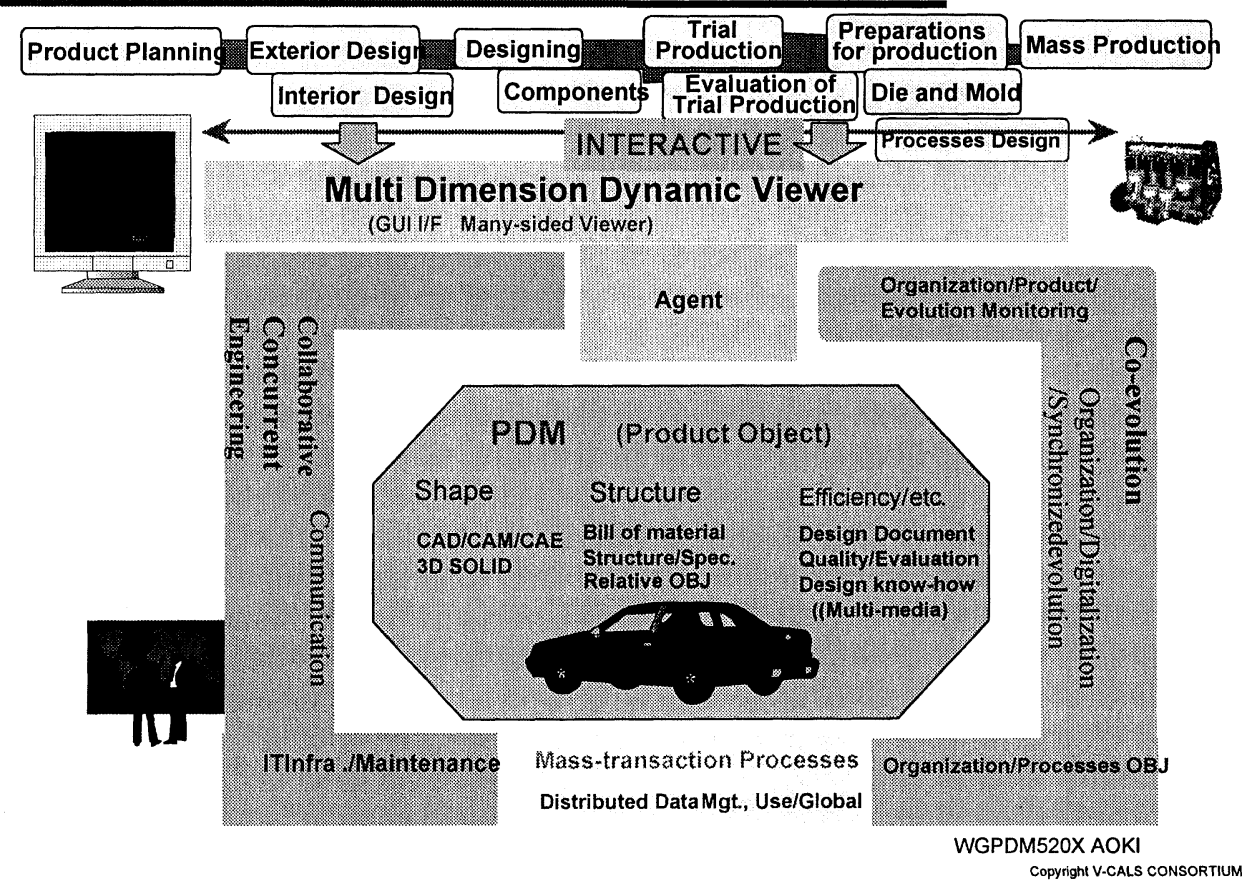

Figure.3 Next-Generation Development Process and PDM research

To respond to the goals of V-CALS, which are to promote standardization and digitalization of product information to enable a response towards a development environment that is becoming more global and more distributed, even more advanced cooperation is required in the new generation of automotive vehicle development. To meet this need, we defined product objects, cooperation design aid, and co-evolution management as basic concepts that will help realize the new generation of automotive development.

In the new generation of automotive development, product objects will be recorded on data bases and shared using CORBA technology. Cooperative design functions that define product objects will be standardized as work objects. Furthermore, by enabling the visualization of product information alongside the development process, a development environment is made in which all people associated with the development can grasp an overview of the purpose and state of development of a product.

In addition, a virtual development space is created in cyberspace by 
digitalization of product objects, work objects, and development process definitions and mutual cooperation is promoted at the same time. This allows for evolution of the motor vehicle development process. In the new generation of automotive development we aim to replace the relic of the past age of mass production where many people were divided into different labor groups, with sharing of product objects using a new generation PDM, and creative automotive development enabled through highly precise engineering that utilizes the help of cooperative design functions.

\subsection{Configuration of Next Generation Vehicle Development System}

After looking at the information and technical requirements for a new generation digital process, we created a four-tiered system configuration as shown in Figure 4. There are four levels, the presentation and application level, the design, object, and service level, the distributed object base, and the database base. As before, there is a distributed object level, which uses CORBA as its key technology, between the application and database levels. It is characterized by the inclusion of a design, object, and service level that acts as a system parts interface group for improving the productivity of future applications.

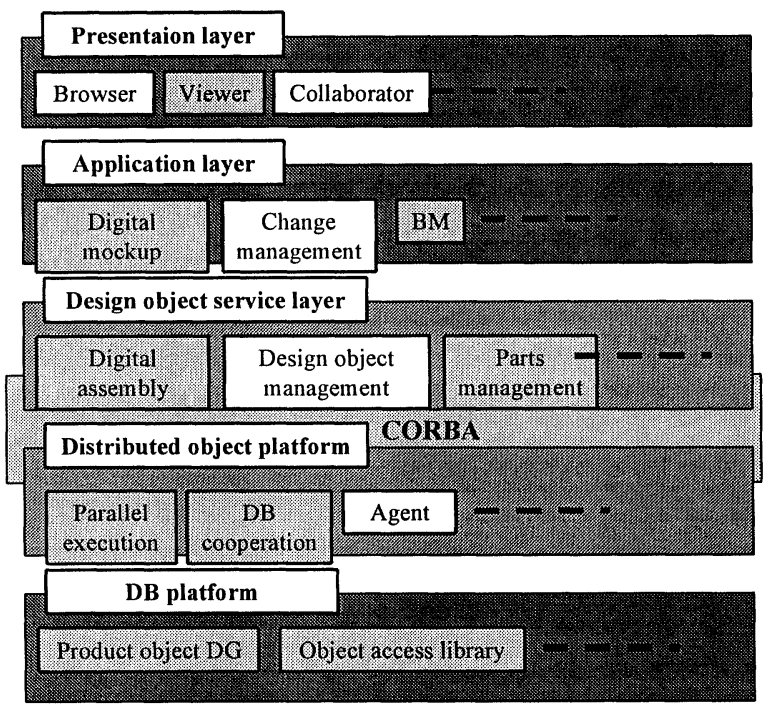

Figure.4 System Configuration

A requirement of a database platform is the ability to enter products into the database. In order to define a database, that supports common vehicle design 
activities, a BPR is required that is capable of organizing an immense amount of data. Figure. 5 shows that the database platform, we are using STEP, AP203, AP214 as a model for implementing the OODB. And we developed an application interface that is comparable to AIM in STEP.

Enter product configuration definitions in DB

Study API that is aware of product configuration informatio

Based on STEP(AP203, 214)
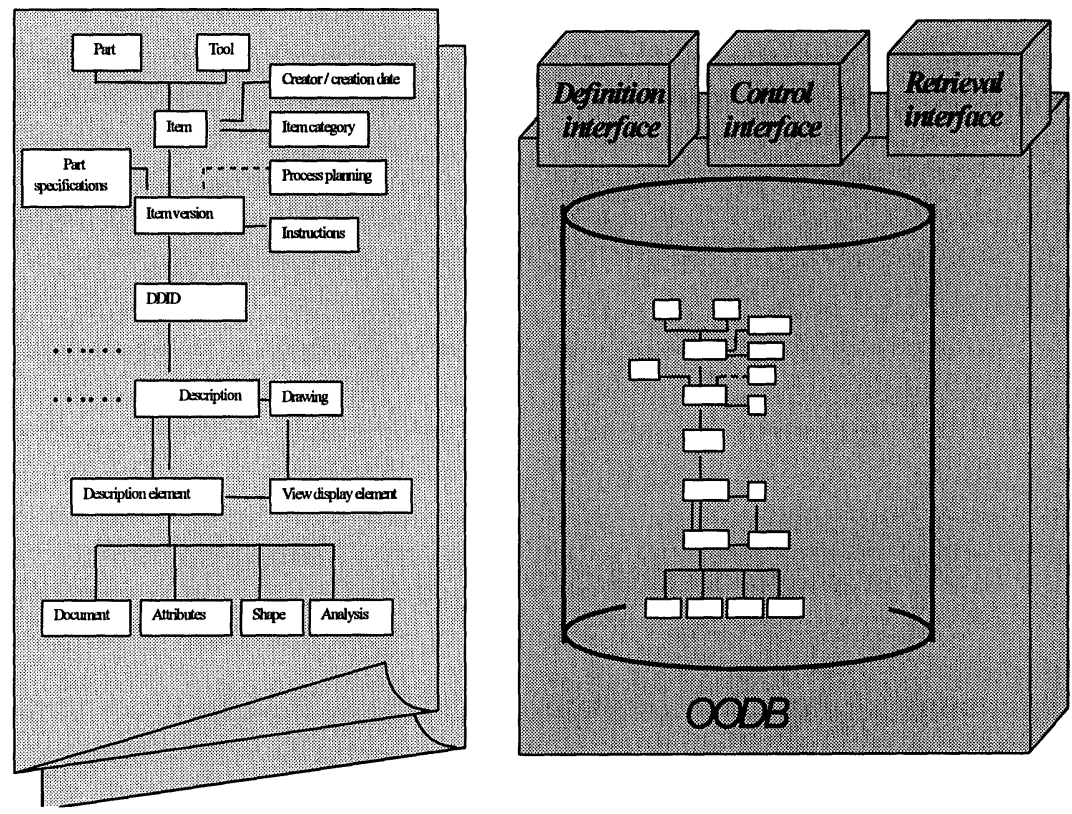

Figure. 5 Product Configuration DB based on STEP

Next trial theme is parallel processing function. This is very effective approach for quickly accessing specific data from a vast sore of information that is scattered on many distributed servers. Since the retrieval object is launched in parallel on the target server, this system will yield dramatically better performance than single 1to- 1 connections.

The third is collaborative concurrent engineering capabilities. Taking the layout of an engine room as an example, complex engine and transmission systems are only the beginning, for body shape data and tires data must also be accessed at the same time.

In order to provide the virtual design support capabilities, we developed a powerful viewer to support design work based on a digital mockup, shown Figure.6. 


\section{Virtualdesign support finction}

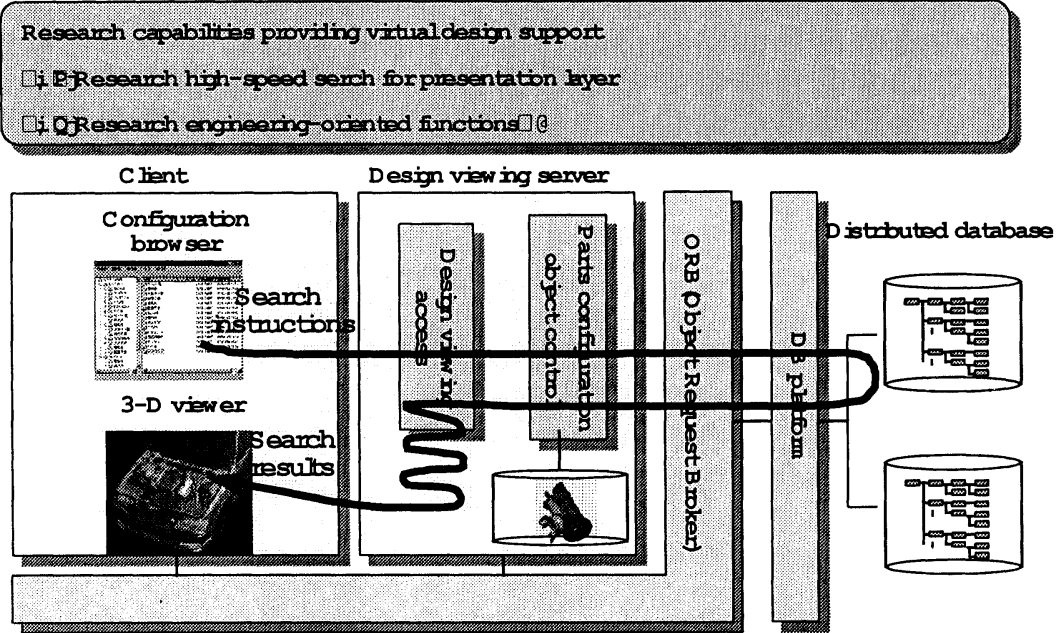

Figure.6 Virtual design support function

The product object will be at the core of next-generation vehicle development, and the tool needed to render the product object. A 3-D browser capable of rendering three-dimensional shapes and a configuration browser that can handle parts configuration data are required. Correspondence between trees and shapes(part recognition) should be achieved quickly by browsing operations illustrated Figure.7. 


\section{Next-Generation Browser}

\section{Engineering functions}

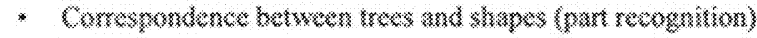

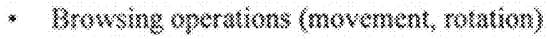

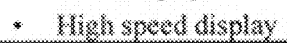

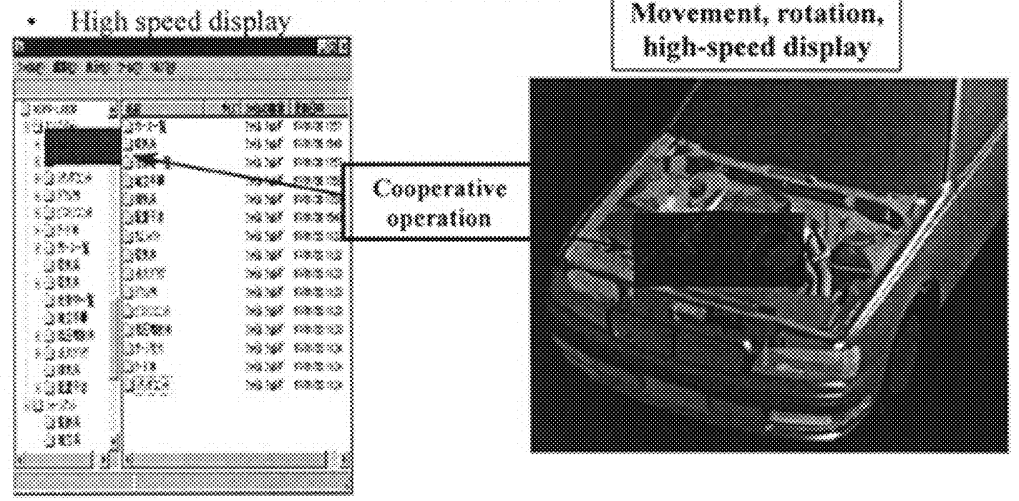

Figure.7 Next-Generation Browser

\subsection{Results of Trials}

In the trials, we tested the "product object search engine functions" and the "cooperative design aid functions" used to test the distributed BM model for motor vehicles around which the database and distributed levels are centered.

(1) Results of trial of product object search engine functions We evaluated the differences in search functions for product objects on the distributed OODB to find the following:

- Differences between concentrated and distributed types

- Total number of parts found

$$
(100,000,500,000,1,000,000)
$$

- Differences in call-back of search results

$$
(1,1,000,5,000,10,000)
$$

- Differences between single search and parallel search

The test patterns can be broadly divided into three groups:

- Product configuration search (following data pointers)

- Simultaneous attribute search using multiple servers

- Detailed measurement of performance for each of above bases (by data base, CORBA, application) 
a) Results of product configuration search

The aim for performance was to operate a simple system within two seconds. The results, including results other than those, were within two seconds, so we are able to say that the goal was achieved. The following can be said after viewing the trends of the results:

- The total number of hits does not depend on performance.

- There is no difference between concentrated and distributed types.

In the single search for product information (search following pointer), the above results exhibited the effectiveness of the OODB

b) Simultaneous search using multiple servers

The results of searches where a part attribute was used as a keyword in a number of servers separately and in parallel are followings.

For operations with a high processing load, we aimed to finish the search in 30 seconds. This goal was achieved for up to 10,000 hits but performance deteriorated when more hits were obtained with the search taking close to 60 seconds. This is a result of the time required for network transmission of a large number of search results rather than a result of search processing performance. In this demonstration we used the 100baseT and 10BaseT but a faster network is required.

In the parallel search, results were equal to approximately $20 \%$ of the single search. In later demonstrations we achieved a further $20 \%$ improvement but this is probably a true indicator of parallel processing.

\section{c) Analysis of performance by level}

The database search performance was greatly affected in each level. In the parallel search, processing was halved because the search processing was distributed and the search load was lightened. The more servers the better the results.

When we view the results above for a), b) and c), all cleared the performance goals and were able to hold up to practical use. As expected, parallel searches were more efficient. However, in the actual field of automotive development design, the environment will be a number of times more complex than the one in the demonstration. An improvement in database access performance and in network speed is desirable.

(2) Results of demonstrations of cooperative design aid functions

a) Details of demonstrations

In relation to the functions developed as above, a demonstration was implemented using actual automotive parts data. The items tested are listed below.

1. Compression rate for form data

2. Conversion time for form data

3. Form display and configuration display time

4. Time required for simple form operation 
5. Single part display time

6. Effect of associative buffer

7. Engineering functions

b) Evaluation and issues raised

We set goals and evaluated these based on the work requirements for the new generation of automotive development.

1. Both data compression rates and conversion times were within the target values so we were able to confirm the effectiveness of this system.

2. Likewise, form display and configuration displays were within the target values and so we were able to confirm the effectiveness of this system. However, the target for simple form operation was not reached. This was not only because of the software. We recognize that there is a need for a major technical revolution in terms of hardware.

3. As can be seen, the associative buffer enabled a great decrease in search times. However, the associated setup needs more research and the associative hit rate needs improvement. We also confirmed the effectiveness of information links between browsers. It will be important in the future to expand the fields of application and create a setup in which designers can appropriate information freely.

\subsection{Summary of SG12}

(1) Adoption of proposal in OMG

In February 1998, results of this study were adopted in the OMG. It was wonderful that these demonstrations were able to test the systems and also contribute to international standards.

(2) The future

The demonstrations only provide a basic setup for realizing a "new generation PDM". In the future we would like to continue investigations at the practical level, and verify practicality and clarify related problems associated with the new generation PDM. The results would be used as guidelines for the future.

\section{OTHER TRIALS AND RESEARCHES}

In this V-CALS project, the demonstrations and research described above were implemented. In addition, the following items were demonstrated, as explained above.

- STEP standardization aids (WG2)

- Standard EDI for motor vehicle industry (WG3) 
- Motor vehicle maintenance information network system (WG4 - SG41)

- Motor vehicle legal and regulation information database system (WG4 SG42)

Because of space limitations, the results of these shall be published at another time.

\section{REVIEW OF V-CALS AND FUTURE PROSPECTS}

The activities of the V-CALS Consortium ended in March 1998, three years after its establishment and after two years of practical work. In this project, we have been able to achieve a great deal. In particular, it is significant that we were able to create an actual prototype, even though imperfect, using the best of the current IT tools.

Through work that attempts to build a bridge between the virtual and the real worlds, we were able to clarify the types of technology, standards, and work reforms that are required for the future.

The Japanese Automobile Manufacture's Association (JAMA) will establish an Electronic Information Committee in April to look at standardization activities. This committee will promote activities that make practical advances in, and that disseminate STEP, EDI, and SGMI in the automotive industry.

A suitable organization that will inherit the results of this practical research and conduct further research in this field is currently under discussion.

I hope that information companies that participated here, will use these results to create a next generation CAD and PDM technology for use throughout the world as commercial software. We really hope that this research will be followed up.

\section{REFERENCES}

1. T.Mase, Vehicle CALS-A big challenge to virtual development, DIISM'96, 1997

2. K.Okamura, A.Arai, and H.Kamba, SG11 : Digital Process Practical Trials, V-CALS consortium, 1998

3. Y.Hori, SG12 : Next-Generation PDM Research, V-CALS consortium, 1998

\section{BIOGRAPHY}

Toshiaki Mase received the B.S degree in mechanical engineering from Kyoto University. He was employed by Nissan Motor Co. LTD. in 1967. He has been working in the area of computer technology and engineering systems development for more than 25years. He managed the projects for developing Nissan and parts venders $\mathrm{CAD} / \mathrm{CAM}$ systems, database systems and styling CAD/CAM system. He was senior manager of body design department in 1990. From 1991 to 1996 he was general manager of Engineering Systems Department Information Systems Division in Nissan. He is currently president of Nissan Digital Process LTD. He had been chairman of V-CALS consortium steering committee from beginning to the end. 